\title{
Cell-Matrix De-Adhesion Dynamics Reflect Contractile Mechanics
}

\author{
Shamik Sen and Sanjay Kumar \\ Department of Bioengineering, University of California, Berkeley, Berkeley, CA 94720-1762, USA
}

(Received 27 February 2009; accepted 16 April 2009; published online 5 May 2009)

\begin{abstract}
Measurement of the mechanical properties of single cells is of increasing interest both from a fundamental cell biological perspective and in the context of disease diagnostics. In this study, we show that tracking cell shape dynamics during trypsin-induced de-adhesion can serve as a simple but extremely useful tool for probing the contractility of adherent cells. When treated with trypsin, both SW13 ${ }^{-/-}$ epithelial cells and U373 MG glioma cells exhibit a brief lag period followed by a concerted retraction to a rounded shape. The time-response of the normalized cell area can be fit to a sigmoidal curve with two characteristic time constants that rise and fall when cells are treated with blebbistatin and nocodazole, respectively. These differences can be attributed to actomyosin-based cytoskeletal remodeling, as evidenced by the prominent buildup of stress fibers in nocodazoletreated SW13 ${ }^{-1-}$ cells, which are also two-fold stiffer than untreated cells. Similar results observed in U373 MG cells highlights the direct association between cell stiffness and the de-adhesion response. Faster de-adhesion is obtained with higher trypsin concentration, with nocodazole treatment further expediting the process and blebbistatin treatment blunting the response. A simple finite element model confirms that faster contraction is achieved with increased stiffness.
\end{abstract}

Keywords-Focal adhesion, Stress fiber, Cytoskeleton, Myosin, Cell mechanics, Atomic force microscopy, Cell stiffness.

\section{ABBREVIATIONS}

$\begin{array}{ll}\text { GBM } & \text { glioblastoma multiforme } \\ \text { ECM } & \text { extracellular matrix } \\ \text { MMP } & \text { matrix metalloprotease } \\ \text { NMMII } & \text { non-muscle myosin II }\end{array}$

\section{INTRODUCTION}

Adhesion of cells to the extracellular matrix (ECM) is key to many aspects of cell physiology including tissue assembly and homeostasis, motility, prolifera-

Address correspondence to Sanjay Kumar, Department of Bioengineering, University of California, Berkeley, Berkeley, CA 947201762, USA. Electronic mail: skumar@berkeley.edu tion, death, and differentiation. The dynamic equilibrium between a cell and its ECM is established through the balance between the contractile forces exerted by the cell and the resistance to deformation offered by the ECM (i.e., ECM rigidity). The cell's ability to sense mechanical cues from the ECM is made possible by engagement and clustering of integrins and formation of focal adhesions, which physically anchor the ECM to the actin cytoskeleton. ${ }^{33}$ Cell mechanobiology involves the study of the mechanisms by which cells sense and respond to mechanical forces, as well as characterization of the mechanical properties of the cell. Cell stiffness or elasticity has emerged as a useful metric for characterizing the mechanical properties of a cell, particularly given that alterations in cell stiffness and/or ECM stiffness often reflect or drive disease progression, as observed in the reduced deformability of malaria-infected red blood cells, ${ }^{23}$ increased stiffness of leukemic cells ${ }^{34}$ and breast tumor tissue, ${ }^{28}$ and enhanced migration and proliferation of glioma cells. ${ }^{46}$ Increases in actomyosin contractility concomitantly increase cell stiffness ${ }^{49}$ and enable cells to stress and remodel the ECM.

The fundamental importance of cell mechanobiology and its increasingly appreciated role in physiology and disease have fueled tremendous interest in developing methods for measuring the mechanical properties of both single cells and populations of cells. Single-cell micromanipulation techniques include micropipet aspiration (MA), ${ }^{6,11,15}$ atomic force microscopy (AFM), ${ }^{34,36,40}$ magnetic bead twisting cytometry (MTC), ${ }^{12,30,47}$ and optical tweezers (OT), ${ }^{1,14,26}$ and typically involve the application of normal or tangential forces in the piconewton $(\mathrm{pN})$ to nanonewton $(\mathrm{nN})$ range. These methods can be utilized for probing individual cells and measuring the elastic or viscoelastic properties of the cell membrane and various subcellular components. In contrast to these single-cell approaches, centrifugation and hydrodynamic assays are used for probing large cell populations and involve the application of either centrifugal forces or shear forces to measure adhesion strength. ${ }^{4,21}$ Recent advances in microfabrication permit the application of 
forces to single or multiple cells with enhanced control of forces and displacements and at significantly higher throughput. For instance, a microfluidic device was recently designed for assessing the deformability of blood cell populations by measuring their transit times through a network of capillaries mimicking blood vessels. ${ }^{35}$ The type of biophysical assay used to probe a particular cell is dictated by its stiffness and the biological process being studied. Although single-cell micromanipulation techniques provide precise measurements of cell mechanical properties, they are lowthroughput and limited by both their high equipment cost and time- and skill intensity. This underscores the need for developing additional simple techniques that do not involve any expensive equipment, are highthroughput, and can measure changes in cell mechanical properties associated with disease or specific physiologic perturbations.

Recent studies have raised the intriguing possibility that the adhesive and de-adhesive dynamics between cells and the ECM may be used to interrogate cellular mechanics. For example, Wildt et al. used a chip-based device composed of RGD-functionalized arrays of microscale gold strips on a glass substrate to study the detachment dynamics of $3 \mathrm{~T} 3$ fibroblasts at the subcellular level. ${ }^{50}$ Rapid release of RGD peptides effected by applying a low voltage pulse across the gold strips led to a corresponding detachment response by the cell characterized by a delay (termed induction time) followed by cell contraction. Both the induction time and the contraction time were shown to depend on cell contractility, with blebbistatin treatment resulting in a progressive increase in both the time scales in a concentration-dependent manner. Similar contractilitydependent retraction dynamics had been reported earlier in the ATP-dependent or trypsin-dependent rounding of endothelial cells, ${ }^{41}$ shape changes in endothelial cells after microneedle-induced cell detachment, ${ }^{29}$ and the relaxation of intact myotubes after mechanical detachment of one end by a glass micropipet. ${ }^{13}$ In all of these studies, the delayed morphological response of cells when treated with actin-disrupting or myosin-inhibiting drugs highlights the role of actomyosin contractility in stressing the ECM and contributing to intracellular tension.

Motivated by the need for high-throughput methods for studying single-cell mechanics and the hint from previous studies of a potential connection between de-adhesion and cellular contractility, we developed a simple and rapid assay to infer cellular mechanical properties from cellular de-adhesive dynamics following protease-mediated detachment from the ECM. Upon treatment with trypsin, both $\mathrm{SW} 13^{-1-}$ epithelial cells and U373 MG glioma cells exhibited a brief lag period followed by a concerted retraction to a rounded shape. The time-response of the normalized cell area could be fit to a sigmoidal curve with two characteristic time constants whose values were sensitive to contractile agonists. By tracking cytoskeletal organization and measuring cortical elasticity by AFM nanoindentation, we correlated changes in retraction kinetics to actomyosin-based remodeling. Further support for the connection between de-adhesive dynamics and cellular mechanics was provided by finite-element simulations, which revealed acceleration of detachment kinetics as cellular elasticity was increased or viscous drag was decreased. Taken together, our results indicate an inverse relationship between the de-adhesion time constants and cell stiffness, and establish the utility of using trypsin-induced de-adhesion as an effective tool for probing cell contractility.

\section{MATERIALS AND METHODS}

\section{Cell Culture}

SW $13^{-/-}$cells were cultured at $37{ }^{\circ} \mathrm{C}$ in a humidified atmosphere of $95 \%$ air and $5 \% \mathrm{CO}_{2}$ in DMEM (Invitrogen) supplemented with 5\% fetal calf serum (J R Scientific, CA). U373 MG human glioblastoma cells (American Type Culture Collection, MD) were cultured under identical conditions in DMEM supplemented with $10 \%$ fetal calf serum, $1 \%$ sodium pyruvate (Invitrogen), 1\% non-essential amino acids (Invitrogen), and 1\% penicillin/streptomycin (Invitrogen). Cells were maintained in $75 \mathrm{~cm}^{2}$ cell culture flasks (Corning, NY), harvested with $0.25 \%$ trypsinEDTA (Invitrogen), and passaged every $2-3$ days. For experiments, cells were plated on glass coverslips coated with $0.05 \mathrm{mg} / \mathrm{mL}$ bovine collagen I (Inamed Biosciences). For de-adhesion studies, cells were incubated with nocodazole or blebbistatin (both from Sigma) or Cytochalasin D (Calbiochem) at the specified concentration for $1 \mathrm{~h}$ before trypsinization (see below).

\section{Immunofluorescence Labeling and Antibodies}

Cultured cells were rinsed twice with phosphatebuffered saline (PBS) (Fisher Scientific), fixed with 4\% para-formaldehyde solution for $10 \mathrm{~min}$, permeabilized with $0.5 \%$ Triton X-100, and blocked in 5\% BSA for $1 \mathrm{~h}$ at room temperature. Cells were incubated for $1 \mathrm{~h}$ at room temperature in one of the following primary antibody solutions in PBS at the specified dilutions: mouse anti-vinculin (1:200, Sigma), mouse antiNMMII (1:200, Santa Cruz). After incubation with primary antibody, cells were rinsed twice with PBS and then incubated with Alexa 543 goat anti-mouse IgG 
(1:500, Invitrogen), and Alexa 488-phalloidin (1:200, Invitrogen) for $1 \mathrm{~h}$ at room temperature. Cell nuclei were labeled with DAPI (1:500, Invitrogen). Samples were mounted onto slides using Cytoseal 60 (RichardAllan Scientific).

\section{Image Acquisition and Analysis}

Both live cell and epifluorescence imaging were performed using a Nikon TE2000E2 microscope equipped with an incubator chamber for controlled temperature, humidity, and $\mathrm{CO}_{2}$. Images were recorded with a CCD camera (Photometrics CoolSNAP HQ2) interfaced to image acquisition software (Compix SimplePCI). For de-adhesion experiments, media was removed, and cells were briefly washed with PBS and then incubated with warm trypsin (either $2.5 \mathrm{~g} / \mathrm{L}$ trypsin with $0.38 \mathrm{~g} / \mathrm{L}$ EDTA (low activity) or $5 \mathrm{~g} / \mathrm{L}$ trypsin with $2 \mathrm{~g} / \mathrm{L}$ EDTA (high activity)). Cells were imaged every 5 or $10 \mathrm{~s}$ at $\times 20$ magnification until cells became rounded with no further apparent change in spread area, and after some delay, eventually detached. The dynamics of de-adhesion were tracked only for the time span when cells were still attached to the substrate. Further, while imaging, the focal plane was set at the cell-substrate interface at the start of the de-adhesion process to ensure that the contact area was being monitored. To quantify de-adhesion, cellsubstrate contact area (subsequently referred to as "cell area") was determined by tracing the outline of the cell at different time points using ImageJ (NIH). The time-dependent normalized area was quantified by dividing the difference between the cell area at time $t$ and the initial spread area (i.e., $A_{\text {initial }}-A(t)$ ) by the difference in area between the first and last time points (i.e., $\left.A_{\text {initial }}-A_{\text {final }}\right)$. Thus, the plot of normalized area increases from a value of 0 (at $\left.t=0, A(t)=A_{\text {initial }}\right)$ to a value of 1 (at $t=t_{\text {final }}, A(t)=A_{\text {final }}$ ). The normalized area-vs.-time data were then fit to a sigmoidal curve to yield the time constants.

\section{Atomic Force Microscopy}

Cells were cultured on glass coverslips as described above. Samples were mounted onto the stage of an Asylum MFP3D AFM (Asylum Research, CA) coupled to a Nikon TE2000E2 epifluorescence microscope and indented using a pyramid-tipped probe (Veeco Instruments) with nominal spring constant of $60 \mathrm{pN} / \mathrm{nm}$. Actual spring constants were determined using the thermal calibration method. Force curves were obtained for 40-50 cells for each condition. Each profile was fit with a modified Hertzian model of a cone indenting a semi-infinite elastic material to extract a set of elastic moduli. ${ }^{36}$
Computational Model of De-Adhesion

An axisymmetric finite element model was developed to simulate de-adhesion dynamics. In this model, cells were compelled to transition from an initially stretched or spread state (S) to a final rounded or relaxed state $(\mathrm{R})$. The cell was modeled as a homogeneous, isotropic, elastic solid with a prescribed Young's modulus $\left(E_{\text {cell }}\right)$, and as undergoing Rayleigh damping with a stiffness damping parameter $(\beta)$. Because perfectly incompressible materials have a Poisson's ratio of 0.5 , and cells are slightly compressible, we fixed the Poisson ratio of the cell at 0.45 for all simulations, which falls well within the range of values used in literature for analysis of AFM indentation measurements. ${ }^{31,39}$ We also performed parameter sensitivity studies, which revealed negligible differences in the simulated curves for Poisson's ratio values between 0.4 and 0.5 . The 'stress-free' rounded state of the cell was modeled as a disk $20 \mu \mathrm{m}$ in height and $20 \mu \mathrm{m}$ in radius. A zero radial displacement boundary condition was imposed on the centerline to ensure symmetry. To isolate effects of contractility, we assumed a frictionless interface between the bottom edge of the cell and the underlying substrate, and that all adhesions were disrupted simultaneously and instantaneously upon addition of trypsin. Therefore, the bottom edge is constrained in $z$-direction but is free to slide in a frictionless manner in the radial direction. The initial state of de-adhesion (i.e., the spread shape) was generated by imposing a prescribed displacement (or stretch) on the outer edge, and solving the static problem, with the stretched state serving as the input for the subsequent de-adhesion simulations. The de-adhesion process was then simulated by imposing free boundary conditions on the outer edge and solving the transient problem. To quantify de-adhesion dynamics, we applied the area normalization scheme outlined above, with the base radius $r(t)$ at any time step converted to an area measured by assuming a circular contact interface. To simulate the effect of contractility and viscosity, we varied the Young's modulus and Rayleigh damping parameter, respectively.

\section{RESULTS}

$$
\text { De-Adhesion Dynamics of SW13-/- Cells }
$$

To quantify cellular de-adhesion dynamics, we developed an assay in which we severed cell-matrix adhesive contacts with trypsin and followed the cellular retraction that precedes cell detachment (Fig. 1a). Our initial studies focused on SW13 $3^{-/-}$cells, an epithelial line that lacks cytoplasmic intermediate filaments $^{38}$ and therefore serves as an excellent model 
(a) $\eta^{t=0: \text { Add trypsin }}$



(b)



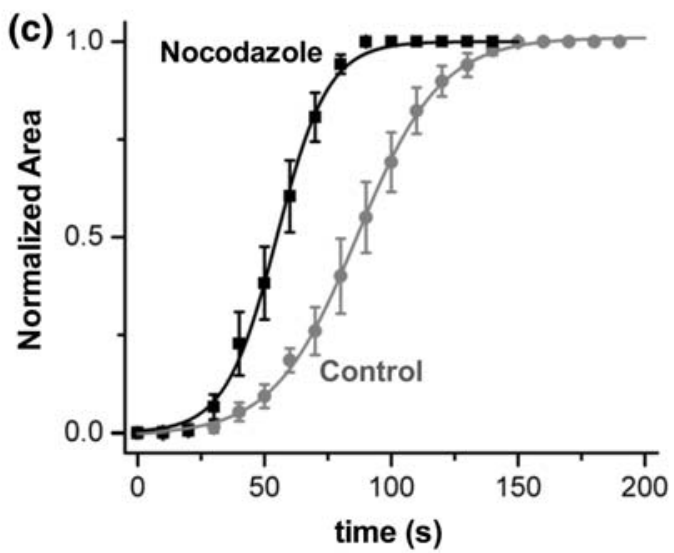

(d)
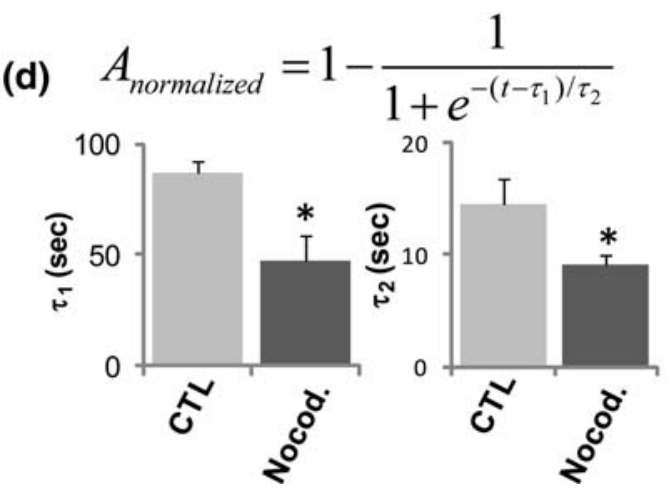

FIGURE 1. De-adhesion dynamics of $\mathrm{SW} 13^{-l-}$ cells. (a) Schematic of trypsin de-adhesion assay. At time $t=0$, media was removed, and cells were briefly washed with PBS and then incubated with warm trypsin. Cells were imaged every 5 or $10 \mathrm{~s}$ at low magnification $(\times 20)$ until cells became rounded. (b) De-adhesion dynamics of untreated (CTL) and $10 \mu \mathrm{M}$ nocodazole-treated (Nocod.) SW13 ${ }^{-l-}$ cells. Scale bar $=90 \mu \mathrm{m}$. (c) Quantification of cell shape changes during de-adhesion. Cell de-adhesion was quantified by plotting the normalized area as a function of time. The detachment response of both control and drug-treated cells was sigmoidal with nocodazole treatment causing faster de-adhesion as observed in the left shift of the sigmoidal curve. (d) Effect of cytoskeletal inhibitors on time constants of retraction. The normalized area-vs.-time data were fit to a Boltzmann sigmoid equation to determine the time constants $\tau_{1}$ and $\tau_{2}$. Faster de-adhesion with nocodazole treatment yielded smaller values for both the time constants $\left({ }^{*} p<0.01\right)$.

system for studying the relative contributions of the actin and microtubule cytoskeletons to cell structure and mechanics. Upon treatment with trypsin, control cells retracted and rounded within 100-150 s, whereas cells treated with the microtubule poison nocodazole retracted and rounded within $50-100 \mathrm{~s}$ (Fig. 1b). To quantify retraction kinetics, we tracked the average normalized cell-substrate contact area (see Methods) as a function of time (Fig. 1c). In both cases, the detachment response of these cells was sigmoidal and composed of three well-defined phases: an initial delay, a rapid cell contraction, and a plateau or saturation. This quantification confirmed our qualitative observations, i.e., whereas control cells took $150 \mathrm{~s}$ to reach
$>95 \%$ saturation, nocodazole-treated cells detached much more quickly and achieved this same level of saturation within $100 \mathrm{~s}$. To further quantify this retraction, we fit each of these curves to a Boltzmann sigmoid equation, which yielded two characteristic time constants, $\tau_{1}$ and $\tau_{2}$ (Fig. 1d). Whereas $\tau_{1} \sim 90 \mathrm{~s}$ for control cells, $\tau_{1} \sim 50 \mathrm{~s}$ for nocodazole-treated cells, which represents a statistically significant difference of $45 \%$. Similarly, nocodazole treatment led to a $40 \%$ reduction in $\tau_{2}(\sim 15 \mathrm{~s}$ and $\sim 9 \mathrm{~s}$ for control and nocodazole-treated and cells, respectively). When cells were treated with the actin-disrupting drug cytochalasin D, cells rapidly collapsed even prior to trypsin treatment (see below) and the de-adhesion process was 
significantly delayed or completely abolished, rendering the retraction data unable to yield time constants (data not shown).

\section{Retraction Dynamics Correlate with Cytoskeletal Architecture and Cell Mechanics in SW13 ${ }^{-1-}$ Cells}

The faster de-adhesion dynamics observed with nocodazole treatment and the ability of cytochalasin D to disrupt this response suggest that these dynamics depend on the structure and mechanics of the actin and microtubule cytoskeletons. To explore this possibility, we visualized the actin and microtubule networks of $\mathrm{SW} 13^{-1-}$ cells in the presence and absence of nocodazole and cytochalasin D. Control cells exhibited thick cortical actin bundles at cell margins and cellcell boundaries but essentially were devoid of welldeveloped stress fibers (Fig. 2a). Disruption of the microtubule network with nocodazole led to a significant reorganization of the actin network, with the emergence of stress fibers spanning the entire length of the cell, consistent with previous observations that nocodazole increases cellular tractional forces. ${ }^{16}$ In contrast, cytochalasin D treatment caused cells to collapse into stellate structures with extended, tubulinpositive membrane protrusions.

The finding that these drug treatments altered both de-adhesion dynamics and cytoskeletal architecture led us to reason that these observations resulted from a common underlying mechanism: altered cellular mechanics. To test this hypothesis directly, we used AFM nanoindentation to measure the elastic moduli of SW13 $3^{-/}$cells in the setting of cytoskeletal inhibition. While control cells were found to have a mean elasticity of $1.6 \mathrm{kPa}$, nocodazole-treated cells were nearly 2 -fold stiffer at a mean stiffness of $3 \mathrm{kPa}$, consistent with the observation that cells develop stress fibers under these conditions. By contrast, cytochalasin D-treated cells were significantly softer at $0.8 \mathrm{kPa}$, representing a $50 \%$ decrease in elasticity compared to control cells. Taken together, these results strongly suggest that the changes in the de-adhesion time constants obtained with nocodazole and cytochalasin D treatment can be explained by an increase in cell stiffness in the treated cells. More specifically, de-adhesion dynamics are inversely correlated with cell stiffness.

\section{De-Adhesion Dynamics of U373 MG Glioma Cells}

While the above observations with $\mathrm{SW} 13^{-/-}$cells suggest that cell stiffness is a critical regulator of de-adhesion dynamics, the interpretation of these results is complicated by the fact that $\mathrm{SW} 13^{-/-}$cells grow in clusters, which may introduce contributions of (a) F-actin


(b)

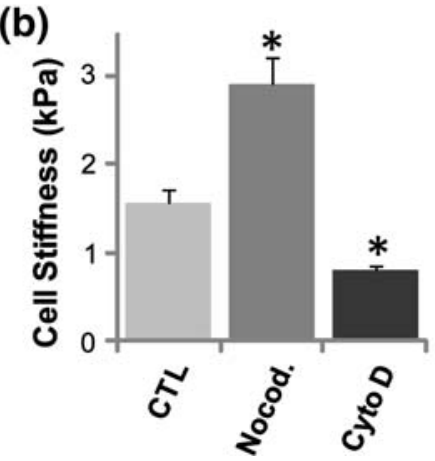

FIGURE 2. Effect of cytoskeletal inhibition on cytoarchitecture and cortical mechanics of SW13 ${ }^{-I-}$ cells. (a) Cytoarchitecture. SW $13^{-l-}$ cells were treated with either microtubule disrupting drug nocodazole $(10 \mu \mathrm{M})$ or actin disrupting drug cytochalasin $\mathrm{D}(0.5 \mu \mathrm{M})$ for $1 \mathrm{~h}$. Subsequent phalloidin staining of F-actin and immunofluorescence against $\beta$-tubulin revealed the redistribution of these two proteins. In contrast to control cells which exhibit thick cortical actin bundles at cell margins and cell-cell boundaries but no stress fibers, nocodazole treatment led to emergence of stress fibers spanning the entire length of the cell. In comparison, cytochalasin D treatment caused cells to collapse into stellate structures with extended, tubulin-positive membrane protrusions. Scale bar $=15 \mu \mathrm{m}$. (b) Cortical cell stiffness. Cortical cell stiffness of control and drug-treated cells was measured with AFM nanoindentation. Compared to controls, drug treatment causes significant differences in cell stiffness ( $\left.{ }^{*} p<0.01\right)$, with nocodazole treatment causing cell stiffening, and cytochalasin $D$ treatment causing cell softening.

cell-cell adhesion and mechanics. To directly evaluate the role of contractility in cells a culture system that lacks these contributions, we repeated the de-adhesion 
experiments with U373 MG glioma cells. U373 MG cells differ from $\mathrm{SW} 13^{-/-}$cells in two important respects: First, when cultured sparsely, U373 MG cells tend to form neither multicellular clusters nor welldeveloped cell-cell adhesive contacts (Fig. 3a). Second, whereas $\mathrm{SW}_{13}{ }^{-1}$ cells adopt a relatively non-polar morphology on collagen coated glass (Fig. 2), U373 MG cells elongate and spread extensively (Fig. 3a). When exposed to trypsin, U373 MG cells began to retract and round within $20 \mathrm{~s}$ and achieved a completely rounded morphology within $60 \mathrm{~s}$ (Fig. 3b). As with the SW13 ${ }^{-/-}$cells, these de-adhesion dynamics followed sigmoidal profiles, which yielded characteristic time constants of $\tau_{1} \sim 26 \mathrm{~s}$ and $\tau_{2} \sim 8 \mathrm{~s}$ (Fig. 3c). Compared to $\mathrm{SW} 13^{--}$cells, this represents a $\sim 67 \%$ reduction in $\tau_{1}$ and a $\sim 50 \%$ reduction in $\tau_{2}$, illustrating that the specific details of the retraction dynamics are cell-type specific. However, like the $\mathrm{SW} 13^{-/-}$cells, nocodazole treatment led to faster de-adhesion with time constants of $\tau_{1} \sim 13 \mathrm{~s}$ and $\tau_{2} \sim 5 \mathrm{~s}$ (Fig. 3c). When we attempted to repeat the cytochalasin D studies (Figs. 1 and 2) with U373 MG cells, we observed that these cells rounded rapidly, did not retract further upon trypsinization, and frequently spontaneously detached, making it challenging for us to either extract time constants or acquire reliable immunostains. Thus, we chose instead to treat these cells with blebbistatin, a non-muscle myosin II (NMMII) inhibitor that dissipates contractility without disassembling the actin cytoskeleton. Under these conditions, we found that cell de-adhesion was significantly delayed, with time constants of $\tau_{1} \sim 45 \mathrm{~s}$ and $\tau_{2} \sim 12 \mathrm{~s}$ (Fig. 3c). Taken together, these results clearly indicate that the de-adhesion dynamics of glioma cells correlate strongly and quantitatively with the mechanical state of the cell.

\section{Retraction Dynamics Correlate with Cytoskeletal Architecture and Cell Mechanics in U373 MG Cells}

Our earlier results with SW13 $3^{-/}$cells (Figs. 1 and 2) demonstrate that faster de-adhesion correlates with both development of cytoskeletal structures associated with increased cortical stiffness. To gain additional mechanistic insight into these changes, we immunostained drug-treated U373 MG cells for vinculin, a marker of mature focal adhesions, and NMMII, the molecular motor whose activity underlies stress fiber contractility (Fig. 4a). Unlike SW13 ${ }^{-/-}$cells, U373 MG cells formed a prominent stress fiber network at baseline and did not develop this network further when treated with nocodazole. However, vinculin staining at focal adhesions was more intense and polarized in nocodazole-treated cells compared to controls. Moreover, NMMII, which is diffusely distributed throughout the cytoplasm in control cells, localized very (a)
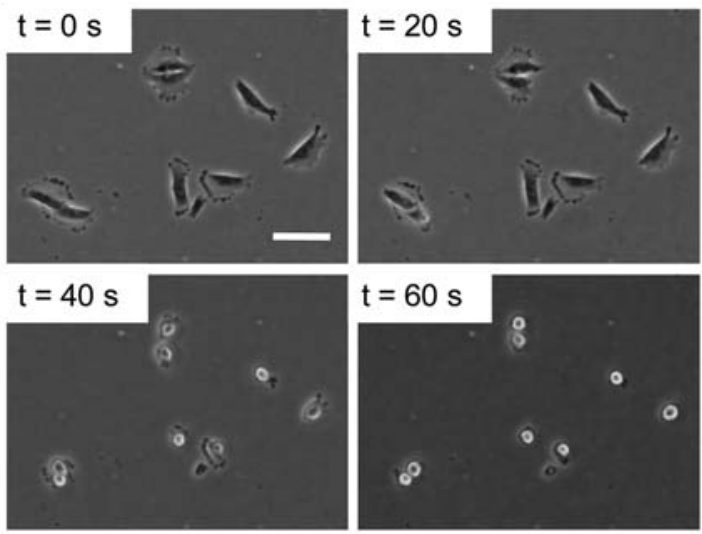

(b)

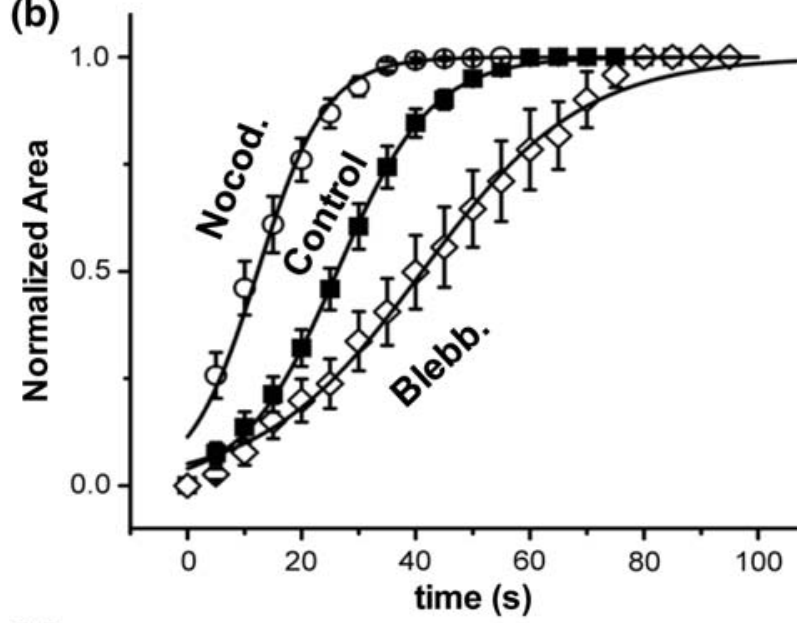

(c)
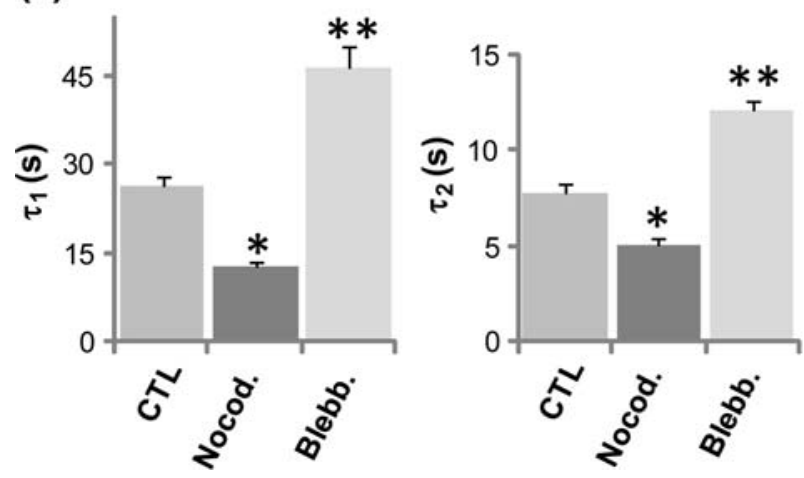

FIGURE 3. De-adhesion dynamics of U373 MG glioma cells. (a) De-adhesion dynamics of individual U373 MG glioma cells. Scale bar $=75 \mu \mathrm{m}$. (b) Quantification of cell shape changes during de-adhesion. Plot of normalized cell area for control, $5 \mu \mathrm{M}$ nocodazole-treated (Nocod.), and $5 \mu \mathrm{M}$ blebbistatintreated (Blebb.) U373 MG cells. While nocodazole treatment caused faster de-adhesion, blebbistatin treatment led to slower de-adhesion. (c) Effect of cytoskeletal inhibitors on time constants of retraction. Differences in $\tau_{1}$ and $\tau_{2}$ were statistically significant for both nocodazole treatment $\left({ }^{*} p<0.1\right)$ and blebbistatin treatment $\left({ }^{*} p<0.001\right)$.

strongly along stress fibers and the cortical actin network (arrowheads), which reflects a more contractile actin cytoskeleton. Treatment with blebbistatin 

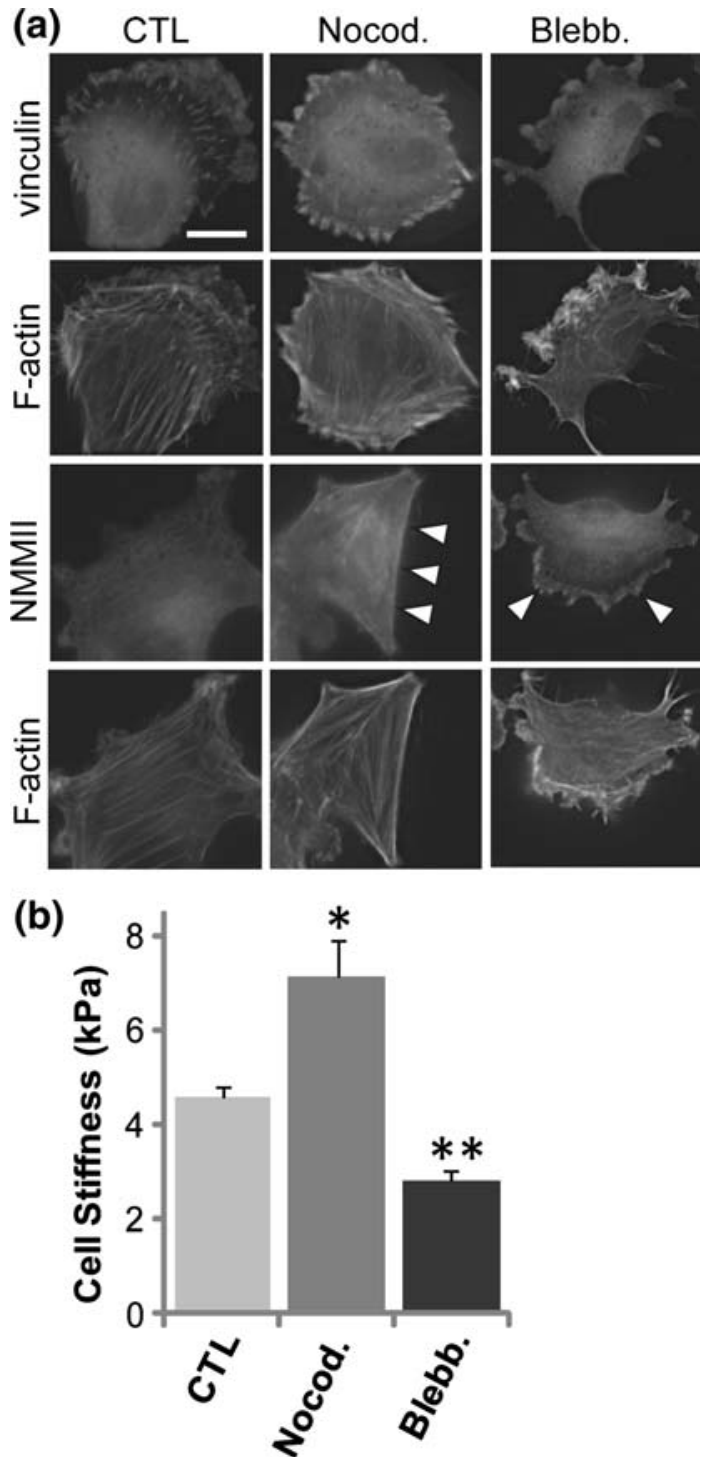

FIGURE 4. Effect of nocodazole and blebbistatin on cytoarchitecture and cortical stiffness in U373 MG cells. (a) Cytoskeletal architecture, focal adhesions, and NMMII localization. U373 MG cells were treated with $5 \mu \mathrm{M}$ nocodazole or $5 \mu \mathrm{M}$ blebbistatin for $1 \mathrm{~h}$ and immunostained for vinculin and NMMII, respectively. Vinculin staining at focal adhesions was more intense and polarized in nocodazole-treated cells compared to controls, but diffuse in blebbistatin-treated cells. NMMII was diffusely distributed in control cells, but localized strongly along stress fibers and cortical actin in nocodazoletreated cells, and was present at membrane ruffles induced by blebbistatin treatment. Scale bar $=15 \mu \mathrm{m}$. (b) Cortical stiffness. Cortical stiffness alterations in drug-treated U373 MG cells. Compared to control cells with a mean stiffness of $\sim 4.5 \mathrm{kPa}$, nocodazole-treated cells were significantly stiffer at $\sim 7 \mathrm{kPa}\left({ }^{*} p<0.01\right)$, and blebbistatin-treated cells were softer at $\sim 2.8 \mathrm{kPa}\left({ }^{* \star} p<0.001\right)$.

completely disassembled the stress fiber network and induced membrane ruffling. While vinculin staining was diffusely distributed throughout the cytoplasm, the membrane ruffles stained strongly for NMMII (arrowheads). AFM measurements confirmed that these drug-induced cytoskeletal alterations were indeed correlated with a more contractile state for the nocodazole-treated cells and a relaxed state for blebbistatin-treated cells. Compared to a mean stiffness of $\sim 4.5 \mathrm{kPa}$ for control cells, nocodazole-treated cells were $\sim 55 \%$ stiffer at $\sim 7 \mathrm{kPa}$ (Fig. $4 \mathrm{~b}$ ). Blebbistatin treatment significantly softened cells and yielded a mean stiffness of $2.8 \mathrm{kPa}$. Taken together, our de-adhesion, immunostaining and AFM results establish a clear connection between cell stiffness and de-adhesion dynamics.

\section{Effect of Trypsin Activity on De-Adhesion}

Our results above establish that changes in de-adhesion dynamics correlate strongly with the development or dissipation of contractile cytoskeletal elements and with cortical stiffness, and that these trends are qualitatively independent of cell-cell contacts. However, it occurred to us that our observed de-adhesion dynamics might also depend on the rate of disruption of cell-substrate adhesions (i.e., trypsin/ EDTA activity), which might vary across drug treatments and cell lines. To directly determine the influence of focal adhesion disassembly on de-adhesion, we repeated our de-adhesion experiments in the presence of cytoskeletal drugs with two different trypsin/EDTA concentrations: $2.5 \mathrm{~g} / \mathrm{L}$ trypsin with $0.38 \mathrm{~g} / \mathrm{L}$ EDTA ("low-activity"), and $5 \mathrm{~g} / \mathrm{L}$ with $2 \mathrm{~g} / \mathrm{L}$ EDTA ("highactivity") (Fig. 5). As expected, for untreated cells, use of the high-activity formulation produced almost instantaneous disruption of focal adhesions relative to the low-activity formulation, with a $\sim 60 \%$ reduction in both of the time constants (Fig. 5a). In nocodazoletreated cells, compared to low-activity trypsin, de-adhesion for high-activity trypsin was further accelerated and led to a $\sim 40 \%$ reduction in $\tau_{1}$ (from 12.66 to $7.6 \mathrm{~s}$ ) and a $\sim 60 \%$ reduction in $\tau_{2}$ (from 5.13 to $2.2 \mathrm{~s}$ ) (Fig. 5b). Interestingly, while high-activity trypsin caused a $\sim 40 \%$ reduction in $\tau_{1}$ in blebbistatin-treated cells relative to $1 \times$ trypsin, $\tau_{2}$ remained unchanged (Fig. 5c). This lack of change could be attributed to the elimination of NMMII-based contractility obtained with blebbistatin treatment, which would indicate that $\tau_{2}$ depends directly on contractility. In contrast, the reduction in $\tau_{1}$ for all the cases indicates that $\tau_{1}$ is modulated by a complex interplay between focal adhesion disassembly and cell contractility.

Another important difference between the de-adhesion curves for low- and high-activity trypsin is that compared to the sigmoidal shape of curves observed with low-activity trypsin, the de-adhesion curves with high-activity trypsin were hyperbolic (saturating) in nature (Fig. 5d). Indeed, these data could be fit with a single exponential representative of viscoelastic relaxation, with $t_{0}$ representing the initial lag and $\tau$ the 
(a)

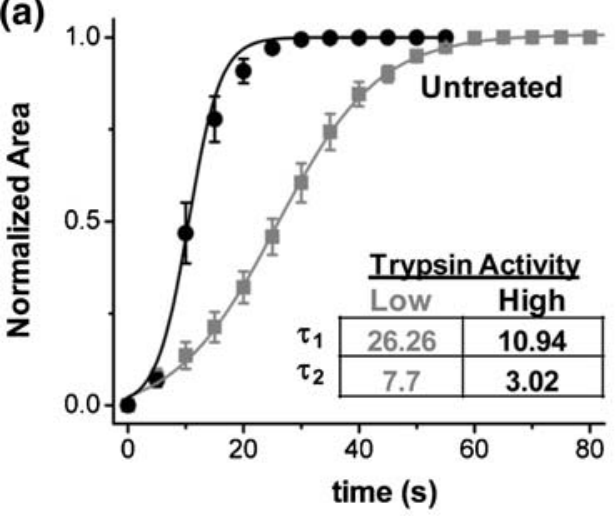

(c)

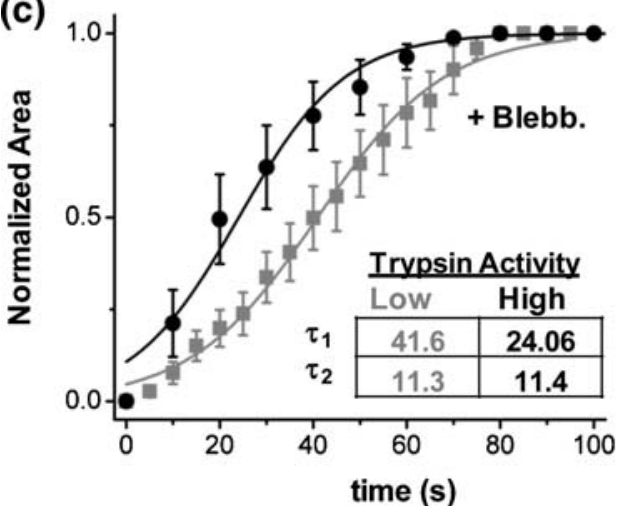

(b)

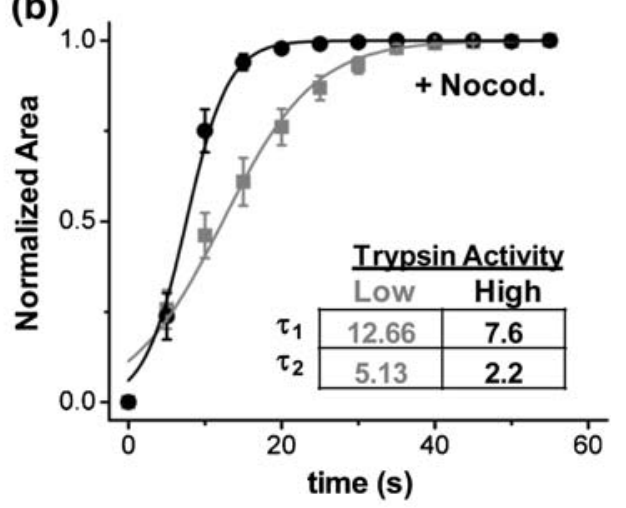

(d)

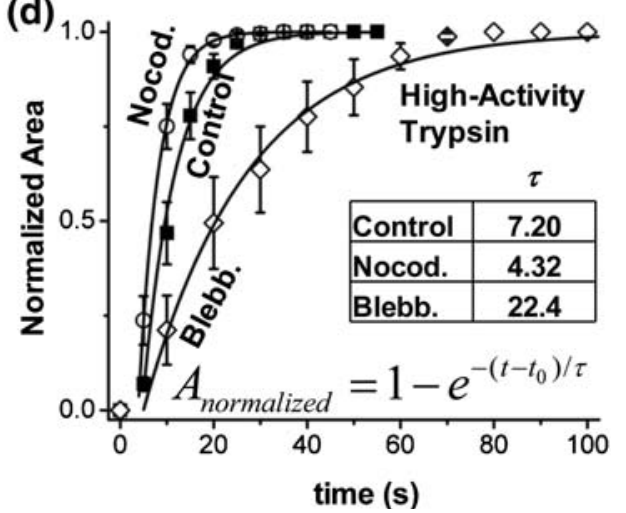

FIGURE 5. Effect of trypsin activity on de-adhesion dynamics of U373 MG cells. (a) De-adhesion dynamics of untreated U373 MG cells exposed to low-activity trypsin and high-activity trypsin, respectively (see Methods). Compared to low-activity trypsin, de-adhesion at high-activity trypsin was faster with a $\sim 60 \%$ reduction in both of the time constants. (b) De-adhesion dynamics of nocodazole-treated U373 MG cells at low- and high-activity trypsin. In nocodazole-treated cells, compared to low-activity trypsin, de-adhesion with high-activity trypsin led to a $\sim 40 \%$ reduction in $\tau_{1}$ and a $\sim 60 \%$ reduction in $\tau_{2}$. (c) De-adhesion dynamics of blebbistatin-treated U373 MG cells at low- and high-activity trypsin. Though high-activity trypsin caused a $\sim 40 \%$ reduction in $\tau_{1}$ in blebbistatin-treated cells relative to low-activity trypsin, $\tau_{2}$ remained unchanged. (d) De-adhesion dynamics of untreated and drugtreated cells at high-activity trypsin fitted with a single exponential representative of viscoelastic relaxation. Compared to controls, the time constant of relaxation $(\tau)$ was lower for nocodazole-treated cells and higher for blebbistatin-treated cells.

characteristic time constant of relaxation. Irrespective of drug treatment, the initial de-adhesion response of both control and drug-treated cells was quite uniform with an initial lag of $4-5 \mathrm{~s}$. The time constant $\tau$ depended on the type of drug treatment with mean values of $\sim 7 \mathrm{~s}$ for control cells, $\sim 4 \mathrm{~s}$ for nocodazoletreated cells, and $\sim 22 \mathrm{~s}$ for blebbistatin-treated cells. A value of $t_{0} \sim 5 \mathrm{~s}$ obtained from these fits suggest that $t_{0}$ is the time required for trypsin to diffuse underneath the cells and disassemble adhesions. Compared to controls, a $\sim 40 \%$ decrease in $\tau$ for nocodazole-treated cells and a $\sim 200 \%$ increase in $\tau$ for blebbistatin-treated cells highlights the role of actomyosin contractility in directly modulating de-adhesion.

\section{Finite Element Modeling of De-Adhesion: Cell Stiffness Directly Modulates De-Adhesion}

The trypsin concentration-dependence data enable us to decouple the effects of contractility and focal adhesion disassembly on de-adhesion dynamics, and highlight the strong influence of contractility in modulating de-adhesion. To further confirm the role of contractility and cytoplasmic viscosity, we simulated de-adhesion in a finite element model in which we could independently vary the elastic modulus of the cell $\left(E_{\text {cell }}\right)$ and the internal damping coefficient $(\beta)$. It is important to note that our model only recapitulates the last phase of de-adhesion dynamics after all adhesions have disassembled. Similar to damped oscillation of a spring-dashpot system, the transient relaxation of our model cell depended directly on $\beta$. While high values of $\beta$ delayed relaxation, as would be expected for an over-damped system, smaller values of $\beta$ led to under-damping, with critical damping obtained in the range of $\beta \sim 1 \times 10^{-5}-5 \times 10^{-5}$. For a cell with prescribed stiffness and $\beta$, different values of imposed initial stretch lead to the same normalized de-adhesion profile (data not shown). We assessed the relationship between cell stiffness and de-adhesion dynamics by 
fixing the damping parameter $\left(2 \times 10^{-5}\right)$ and varying the cell stiffness over 3 orders of magnitude from 0.1 to $10 \mathrm{kPa}$ (Fig. 6b). At $0.1 \mathrm{kPa}$, de-adhesion was found to be almost linear indicating little or no change in area. Increasing stiffness led to faster de-adhesion as observed in the left shift of the curves $(2$ and $10 \mathrm{kPa}$,

(a)


FIGURE 6. Finite element model of de-adhesion: effect of cell stiffness. (a) Schematic comparison of cell spreading and cell de-adhesion. For studying de-adhesion dynamics, an initial spread shape (S) was generated by stretching the model disk-shaped cell $(R)$ representative of the final rounded state observed experimentally. De-adhesion was simulated by allowing the stretched cell to relax and solving the transient problem. The simulation parameters include the damping parameter $(\beta)$ and the cell stiffness $\left(E_{\text {cell }}\right)$. Displacement map induced by stretching the initially relaxed cell was plotted, with red being the maximum displacement and blue being zero displacement. This stretched state served as the starting cell configuration for solving the transient problem. (b) Simulated de-adhesion curves obtained for $\beta=2 \times 10^{-5}$ and $E_{\text {cell }}$ $(\mathrm{kPa})=0.1,2.0$, and 10.0 , respectively. respectively). The overshoot observed at $2 \mathrm{kPa}$ indicates the system is under-damped for the choice of stiffness and $\beta$. The fastest dynamics observed at $10 \mathrm{kPa}$ establishes the direct influence of cell stiffness in modulating de-adhesion.

\section{DISCUSSION}

In this study, we have demonstrated that trypsininduced de-adhesion can be used as a simple yet effective tool for probing the contractility of adherent cells. We found that when treated with trypsin, adherent cells detach and round with sigmoidal dynamics. The time constants of this process are sensitive to cytoskeletal inhibitors, with nocodazole and blebbistatin treatment accelerating and delaying de-adhesion, respectively. Contractility-dependent alterations of these time constants closely mirror alterations in the stiffness of drug-treated cells, with nocodazole and blebbistatin treatment significantly enhancing and lowering cell stiffness, respectively. The observation that the de-adhesion dynamics also depend strongly on the activity of the trypsin formulation highlights the importance of proteolysis and disassembly of cell-matrix adhesions in regulating the initial phase of de-adhesion. Finally, we capture the modulation of de-adhesion by contractility in a simple finite element model in which increased cell stiffness leads to faster de-adhesion. Our results thus demonstrate that de-adhesion time constants scale inversely with cell contractility and stiffness and establish trypsin-induced de-adhesion as a simple tool for probing the contractility of adherent cells. We anticipate that this approach may serve as a semiquantitative, highthroughput complement to single-cell micromechanical approaches (e.g., AFM, MA, OT), which measure cell mechanical properties more precisely and directly but are also both low-throughput and labor- and skillintensive. By combining motorized microscopy platforms and commercially available cell tracking software, we anticipate that the method can be automated and made suitable for high-throughput optical analysis of cell contractility. Furthermore, the simplicity of our technique allows it to be easily integrated into other high-throughput techniques for cellular analysis. For example, we envision that cells might be sorted according to their de-adhesion kinetics and delivered to a flow cytometry system to correlate micromechanics to expression of specific markers. Given the emerging evidence that altered cellular mechanics may serve as a biomarker for stem cell differentiation $^{10,37}$ and disease states, ${ }^{23,28,34}$ such a system could prove extremely valuable for bioprocessing and diagnostic applications. 
For both SW13 ${ }^{-1-}$ and U373 MG cells, the pharmacologically induced alterations in cell contractility led to clear and significant changes in the cortical stiffness of cells by AFM. The close association between cell prestress (or contractile mechanics) and stiffness (cortical mechanics) in adherent cells has been previously demonstrated in human airway smooth muscle cells ${ }^{44}$; when cell contractility was modulated through the use of contractile agonists and antagonists, cellular traction forces scaled linearly with cell stiffness by MTC. This association between contractility and stiffness has also been observed in the context of the adaptation of fibroblasts to ECM stiffness. ${ }^{42}$ Specifically, fibroblasts match the stiffness of their underlying ECM substrate for ECM stiffnesses up to $5 \mathrm{kPa}$ and do so without forming stress fibers. At greater ECM stiffnesses, cell stiffness plateaus and cells form stress fibers, because they are unable to reinforce their adhesions further simply by reorganizing the cortical actin network. Analogous to this, stress fibers are observed in $\mathrm{SW}_{1} 3^{-/-}$cells only when contractility is enhanced by nocodazole treatment.

The significant decrease in the stiffness of cytochalasin D-treated SW13 ${ }^{-/-}$cells observed here and elsewhere ${ }^{25,45}$ demonstrate the dominance of F-actin in determining the stiffness of cells. In addition, the significant increase in cell stiffness obtained with nocodazole treatment is consistent with the tensegrity model. ${ }^{17,18}$ Increased traction in response to microtubule disruption has been reported in various studies and highlight the reciprocal role for actin and microtubules. $5,20,43,48$ This has also been observed in $3 \mathrm{D}$ cultures in the enhanced retraction of microtubuledisrupted smooth muscle cells in a quasi-in situ tensile test. ${ }^{24}$ Increased myosin phosphorylation ${ }^{19}$ and high intracellular calcium ${ }^{27}$ observed upon microtubule disruption may directly contribute to the enhanced stiffness of nocodazole-treated cells observed here.

By examining multiple cell lines (SW13 ${ }^{-1-}$ and U373 MG), we were able to demonstrate the generality of the method while also observing characteristic cellspecific differences in retraction dynamics. While $\mathrm{SW} 13^{-/-}$cells grow in clusters and are rounded in shape, U373 MG cells spread extensively, and have a more strongly developed stress fiber network. Consequently, in comparison to a baseline stiffness of $4.5 \mathrm{kPa}$ measured for U373 MG cells, SW13 ${ }^{-/-}$cells are $\sim 60 \%$ softer at $1.6 \mathrm{kPa}$. The emergence of stress fibers upon nocodazole treatment in $\mathrm{SW} 13^{-1-}$ cells but not in U373 MG cells further can be attributed to the lower contractile state of SW13 $3^{-/}$cells. Furthermore, compared to $\mathrm{SW} 13^{-/-}$cells, a $\sim 67 \%$ reduction in $\tau_{1}$ and a $\sim 50 \%$ reduction in $\tau_{2}$ of U373 MG cells reflects the higher baseline contractility of U373 MG cells and illustrates the cell-type specific differences in the retraction dynamics. Even in blebbistatin-treated U373 MG cells, $\tau_{1} \sim 45 \mathrm{~s}$, which is still $\sim 50 \%$ faster than control SW $13^{-/-}$cells. Moreover, while blebbistatintreated U373 MG cells are softer than untreated U373 MG cells, they have a mean stiffness of $2.8 \mathrm{kPa}$, which is $\sim 75 \%$ stiffer than untreated $\mathrm{SW}_{1} 3^{-/}$cells. In addition to highlighting cell-type differences in baseline contractility, these results demonstrate that both cell stiffness and de-adhesion reflect the contractile state of the cell. From our experimental data, cell stiffness and de-adhesion time constants of SW13 ${ }^{-1-}$ and U373 cells can be combined to obtain approximate expressions $\left(E_{\text {cell }} \approx 54 \tau_{1}^{-0.775}\left(R^{2}=0.99\right)\right.$ and $E_{\text {cell }} \approx$ $64 \tau_{2}^{-1.336}\left(R^{2}=0.94\right)$, respectively) which explicitly relate the cell stiffness to the two time constants. In addition to providing a method to estimate cell stiffness, these expressions reinforce the inverse correlation between stiffness and de-adhesion time constants.

The strong dependence of the detachment dynamics on trypsin/EDTA activity reveals insight into the contributions of contractility and focal adhesion disassembly to de-adhesion dynamics. These studies suggest that one time constant reflects both adhesion disassembly and contractility, whereas the other time constant primarily reflects contractility, a notion that is further supported by our simulated de-adhesion curves. Also, as expected, de-adhesion depends directly on cytoplasmic viscosity, represented in our simulations by the damping coefficient. While this exceedingly simple model yields valuable mechanistic insight into de-adhesion dynamics, much room remains for additional sophistication. For example, our simulations model only the second phase of high-activity trypsin experiments, i.e., they assume instantaneous breakage of all adhesions and frictionless sliding of the cell on the substrate dictated solely by cell stiffness. Incorporation of position- or time-dependent trypsin activity may more faithfully recreate the experimental de-adhesion curves. Additionally, the model would likely benefit from a more realistic representation of cell geometry and cytoarchitecture, including more explicit description of cytoskeletal filaments and assignation of different mechanical properties to the individual components, and inclusion of friction between the cell surface and the substrate. More importantly, the temporal effects of focal adhesion disassembly and contractility on de-adhesion dynamics can be decoupled by incorporating discrete adhesions in our simulations. The NMMII isoform NMMIIA preferentially affects the stability of focal adhesions, ${ }^{3}$ suggesting that the interplay between adhesion and contractility may be better captured by allowing the discrete adhesions to disassemble in a stress- or straindependent manner. 
In traction force microscopy, cellular traction forces are determined by measuring the displacements of matrix-embedded beads after dissipation of matrix strains achieved by physically detaching the cell from the substrate. ${ }^{2}$ Recently, tractional stresses and strains associated with discrete contractile structures were measured by using laser ablation to sever a single stress fiber while simultaneously tracking bead displacements in the underlying substrate. ${ }^{22}$ In this study, traction force relaxation followed saturation kinetics with a plateau achieved within $10 \mathrm{~s}$ of ablation. The time scale of relaxation in this study compares well with the time scale of detachment observed with U373 MG cells. The dependence of the de-adhesion time constants on cell contractility suggests that they are directly related to traction forces and may potentially have value as a surrogate for directly monitoring the time-dependent relaxation of traction forces and matrix strains, which is a significantly more time- and labor-intensive experiment.

Cell motility requires the coordinated extension of membrane protrusions, formation of transient adhesions, and myosin-dependent detachment of the cell rear. ${ }^{33}$ Therefore, precise synchronization of adhesion at the leading edge and de-adhesion at the rear are crucial to motility to occur. Interestingly, a comparison of the de-adhesion dynamics observed in our studies with adhesion dynamics of endothelial cells ${ }^{32}$ reveals surprising similarities between the two processes. Both processes are marked by an initial lag followed by rapid change in cell-substrate contact area and an eventual saturation. Similar adhesion dynamics have also been observed in the spreading of mouse embryonic fibroblasts on fibronectin-coated substrates, and reveal phase transitions during cell spreading. ${ }^{7}$ However, the time scales of these two processes are different, with de-adhesion occurring within seconds, and adhesion occurring in tens of minutes. Also, while the lag phase during spreading depends on ligand density, ${ }^{8,32}$ the lag phase during de-adhesion is related to trypsin diffusion times. However, the increase in cell-exerted traction forces during spreading, ${ }^{9,32}$ and the delayed de-adhesion response of blebbistatintreated cells illustrate the role of myosin in modulating both these processes.

In conclusion, we have developed a simple assay to determine cell mechanical properties from de-adhesive dynamics following trypsin-induced detachment and shown that de-adhesion time constants correlate inversely with cell contractility or stiffness. The sensitivity of our assay allows us to detect differences in cell mechanics associated with changes in cell stiffness of less than $1 \mathrm{kPa}$ and makes our assay a useful tool for studying induced differences in mechanical properties for a single cell type, as well as variations in cell mechanics across multiple cell types. Furthermore, by manipulating the trypsin concentration, our method can be adapted to explore the interplay between adhesion and contractility. We anticipate that the simplicity of our technique will make it particularly suitable for integration with high-throughput analytical techniques for use in diagnostic and bioprocessing applications.

\section{ACKNOWLEDGMENTS}

S.K. gratefully acknowledges the support of the University of California, the Arnold and Mabel Beckman Young Investigator Award, and the NIH Director's New Innovator Award (1DP2OD004213), a part of the NIH Roadmap for Medical Research. We would also like to thank Dr. Robert Evans (University of Colorado) for kindly providing the $\mathrm{SW} 13^{-/-}$cells.

\section{OPEN ACCESS}

This article is distributed under the terms of the Creative Commons Attribution Noncommercial License which permits any noncommercial use, distribution, and reproduction in any medium, provided the original author(s) and source are credited.

\section{REFERENCES}

${ }^{1}$ Ashkin, A., J. M. Dziedzic, and T. Yamane. Optical trapping and manipulation of single cells using infrared laser beams. Nature 330(6150):769-771, 1987.

${ }^{2}$ Butler, J. P., I. M. Tolic-Norrelykke, B. Fabry, and J. J. Fredberg. Traction fields, moments, and strain energy that cells exert on their surroundings. Am. J. Physiol. Cell Physiol. 282(3):C595-C605, 2002.

${ }^{3}$ Cai, Y., N. Biais, G. Giannone, et al. Nonmuscle myosin IIA-dependent force inhibits cell spreading and drives F-actin flow. Biophys. J. 91(10):3907-3920, 2006.

${ }^{4}$ Catherine, D., and A. García. A centrifugation cell adhesion assay for high-throughput screening of biomaterial surfaces. J. Biomed. Mater. Res. A 67(1):328-333, 2003.

${ }^{5}$ Danowski, B. Fibroblast contractility and actin organization are stimulated by microtubule inhibitors. J. Cell Sci. 93(2):255-266, 1989.

${ }^{6}$ Discher, D. E., D. H. Boal, and S. K. Boey. Simulations of the erythrocyte cytoskeleton at large deformation. II. Micropipette aspiration. Biophys. J. 75(3):1584-1597, 1998. ${ }^{7}$ Dobereiner, H. G., B. Dubin-Thaler, G. Giannone, H. S. Xenias, and M. P. Sheetz. Dynamic phase transitions in cell spreading. Phys. Rev. Lett. 93(10):108105, 2004.

${ }^{8}$ Dubin-Thaler, B. J., G. Giannone, H. G. Dobereiner, and M. P. Sheetz. Nanometer analysis of cell spreading on matrix-coated surfaces reveals two distinct cell states and STEPs. Biophys. J. 86(3):1794-1806, 2004. 
${ }^{9}$ Dubin-Thaler, B. J., J. M. Hofman, Y. Cai, et al. Quantification of cell edge velocities and traction forces reveals distinct motility modules during cell spreading. PLOS ONE 3(11):e3735, 2008.

${ }^{10}$ Engler, A. J., S. Sen, H. L. Sweeney, and D. E. Discher. Matrix elasticity directs stem cell lineage specification. Cell 126(4):677-689, 2006.

${ }^{11}$ Evans, E., and A. Yeung. Apparent viscosity and cortical tension of blood granulocytes determined by micropipet aspiration. Biophys. J. 56(1):151-160, 1989.

${ }^{12}$ Fabry, B., G. N. Maksym, S. A. Shore, et al. Signal transduction in smooth muscle: selected contribution: time course and heterogeneity of contractile responses in cultured human airway smooth muscle cells. J. Appl. Physiol. 91(2):986-994, 2001.

${ }^{13}$ Griffin, M. A., S. Sen, H. L. Sweeney, and D. E. Discher. Adhesion-contractile balance in myocyte differentiation. J. Cell Sci. 117(24):5855-5863, 2004.

${ }^{14}$ Hénon, S., G. Lenormand, A. Richert, and F. Gallet. A new determination of the shear modulus of the human erythrocyte membrane using optical tweezers. Biophys. J. 76(2):1145-1151, 1999.

${ }^{15}$ Hochmuth, R. M. Micropipette aspiration of living cells. J. Biomech. 33(1):15-22, 2000.

${ }^{16} \mathrm{Hu}, \mathrm{S}$., J. Chen, and N. Wang. Cell spreading controls balance of prestress by microtubules and extracellular matrix. Front. Biosci. 9:2177-2182, 2004.

${ }^{17}$ Ingber, D. Cellular tensegrity: defining new rules of biological design that govern the cytoskeleton. J. Cell Sci. 104(3):613-627, 1993

${ }^{18}$ Ingber, D. E. Tensegrity. I. Cell structure and hierarchical systems biology. J. Cell Sci. 116(7):1157-1173, 2003.

${ }^{19}$ Kolodney, M. S., and E. L. Elson. Contraction due to microtubule disruption is associated with increased phosphorylation of myosin regulatory light chain. Proc. Natl. Acad. Sci. USA 92(22):10252-10256, 1995.

${ }^{20}$ Kolodney, M., and R. Wysolmerski. Isometric contraction by fibroblasts and endothelial cells in tissue culture: a quantitative study. J. Cell Biol. 117(1):73-82, 1992.

${ }^{21}$ Konstantopoulos, K., S. Kukreti, and L. V. McIntire. Biomechanics of cell interactions in shear fields. Adv. Drug Deliv. Rev. 33(1-2):141-164, 1998.

${ }^{22}$ Kumar, S., I. Z. Maxwell, A. Heisterkamp, et al. Viscoelastic retraction of single living stress fibers and its impact on cell shape, cytoskeletal organization, and extracellular matrix mechanics. Biophys. J. 90(10):3762-3773, 2006.

${ }^{23}$ Marinkovic, M., M. Diez-Silva, I. Pantic, J. J. Fredberg, S. Suresh, and J. P. Butler. Febrile temperature leads to significant stiffening of Plasmodium falciparum parasitized erythrocytes. Am. J. Physiol. Cell Physiol. 296(1):C59-C64, 2009.

${ }^{24}$ Nagayama, K., and T. Matsumoto. Contribution of actin filaments and microtubules to quasi-in situ tensile properties and internal force balance of cultured smooth muscle cells on a substrate. Am. J. Physiol. Cell Physiol. 295(6):C1569-C1578, 2008

${ }^{25}$ Nagayama, K., Y. Nagano, M. Sato, and T. Matsumoto. Effect of actin filament distribution on tensile properties of smooth muscle cells obtained from rat thoracic aortas. J. Biomech. 39(2):293-301, 2006.

${ }^{26}$ Neuman, K. C., and A. Nagy. Single-molecule force spectroscopy: optical tweezers, magnetic tweezers and atomic force microscopy. Nat. Methods 5(6):491-505, 2008.

${ }^{27}$ Obara, K., K. Nobe, H. Nobe, M. S. Kolodney, P. De Lanerolle, and R. J. Paul. Effects of microtubules and microfilaments on $[\mathrm{Ca} 2+] \mathrm{i}$ and contractility in a reconstituted fibroblast fiber. Am. J. Physiol. Cell Physiol. 279(3): C785-C796, 2000.

${ }^{28}$ Paszek, M. J., N. Zahir, K. R. Johnson, et al. Tensional homeostasis and the malignant phenotype. Cancer Cell 8(3):241-254, 2005.

${ }^{29}$ Pourati, J., A. Maniotis, D. Spiegel, et al. Is cytoskeletal tension a major determinant of cell deformability in adherent endothelial cells? Am. J. Physiol. Cell Physiol. 274(5):C1283-C1289, 1998.

${ }^{30}$ Puig-De-Morales, M., M. Grabulosa, J. Alcaraz, et al. Measurement of cell microrheology by magnetic twisting cytometry with frequency domain demodulation. J. Appl. Physiol. 91(3):1152-1159, 2001.

${ }^{31}$ Radmacher, M. Studying the mechanics of cellular processes by atomic force microscopy. Methods Cell Biol. 83:347-372, 2007.

${ }^{32}$ Reinhart-King, C. A., M. Dembo, and D. A. Hammer. The dynamics and mechanics of endothelial cell spreading. Biophys. J. 89(1):676-689, 2005.

${ }^{33}$ Ridley, A. J., M. A. Schwartz, K. Burridge, et al. Cell migration: integrating signals from front to back. Science 302(5651):1704-1709, 2003.

${ }^{34}$ Rosenbluth, M. J., W. A. Lam, and D. A. Fletcher. Force microscopy of nonadherent cells: a comparison of leukemia cell deformability. Biophys. J. 90(8):2994-3003, 2006.

${ }^{35}$ Rosenbluth, M. J., W. A. Lam, and D. A. Fletcher. Analyzing cell mechanics in hematologic diseases with microfluidic biophysical flow cytometry. Lab Chip 8(7): 1062-1070, 2008.

${ }^{36}$ Rotsch, C., K. Jacobson, and M. Radmacher. Dimensional and mechanical dynamics of active and stable edges in motile fibroblasts investigated by using atomic force microscopy. Proc. Natl. Acad. Sci. USA 96(3):921-926, 1999.

${ }^{37}$ Saha, K., A. J. Keung, E. F. Irwin, et al. Substrate modulus directs neural stem cell behavior. Biophys. J. 95(9):44264438, 2008.

${ }^{38}$ Sarria, A., J. Lieber, S. Nordeen, and R. Evans. The presence or absence of a vimentin-type intermediate filament network affects the shape of the nucleus in human SW-13 cells. J. Cell Sci. 107(6):1593-1607, 1994.

${ }^{39}$ Schachar RA. Determination of the Poisson's ratio of the cell: recovery properties of chondrocytes after release from complete micropipette aspiration (Trickey et al., J. Biomech. 39 (2006) 78-87). J. Biomech. 39(12):2344, 2006; author reply 2344-2345.

${ }^{40}$ Sen, S., S. Subramanian, and D. E. Discher. Indentation and adhesive probing of a cell membrane with AFM: theoretical model and experiments. Biophys. J. 89(5):3203$3213,2005$.

${ }^{41}$ Sims, J., S. Karp, and D. Ingber. Altering the cellular mechanical force balance results in integrated changes in cell, cytoskeletal and nuclear shape. J. Cell Sci. 103(4): 1215-1222, 1992.

${ }^{42}$ Solon, J., I. Levental, K. Sengupta, P. C. Georges, and P. A. Janmey. Fibroblast adaptation and stiffness matching to soft elastic substrates. Biophys. J. 93(12):4453-4461, 2007.

${ }^{43}$ Stamenovic, D., S. M. Mijailovich, I. M. Tolic-Norrelykke, J. Chen, and N. Wang. Cell prestress. II. Contribution of microtubules. Am. J. Physiol. Cell Physiol. 282(3):C617C624, 2002

${ }^{44}$ Thoumine, O., and A. Ott. Time scale dependent viscoelastic and contractile regimes in fibroblasts probed by microplate manipulation. J. Cell Sci. 110(17):2109-2116, 1997. 
${ }^{45}$ Trickey, W., T. Vail, and F. Guilak. The role of the cytoskeleton in the viscoelastic properties of human articular chondrocytes. J. Orthop. Res. 22(1):131-139, 2004.

${ }^{46}$ Ulrich, T. A., E. M. de Juan Pardo, and S. Kumar. The mechanical rigidity of the extracellular matrix regulates the structure, motility, and proliferation of glioma cells. Cancer Res. 2009, in press.

${ }^{47}$ Wang, N., J. Butler, and D. Ingber. Mechanotransduction across the cell surface and through the cytoskeleton. Science 260(5111):1124-1127, 1993.
${ }^{48}$ Wang, N., K. Naruse, D. Stamenovic, et al. Mechanical behavior in living cells consistent with the tensegrity model. Proc. Natl. Acad. Sci. USA 98(14):7765-7770, 2001.

${ }^{49}$ Wang, N., I. M. Tolic-Norrelykke, J. Chen, et al. Cell prestress. I. Stiffness and prestress are closely associated in adherent contractile cells. Am. J. Physiol. Cell Physiol. 282(3):C606-C616, 2002.

${ }^{50}$ Wildt, B., D. Wirtz, and P. C. Searson. Programmed subcellular release for studying the dynamics of cell detachment. Nat. Methods 6(3):211-213, 2009. 